\title{
Intracranial meningeal hemangiopericytoma: Recurrences at the initial and distant intracranial sites and extraneural metastases to multiple organs
}

\author{
GUANGQUAN WEI ${ }^{1}$, XIAOWEI KANG ${ }^{1}$, XIANPING LIU ${ }^{1}$, XING TANG $^{1}$, \\ QINLONG LI ${ }^{2}$, JUNTAO HAN ${ }^{3}$ and HONG YIN ${ }^{1}$ \\ Departments of ${ }^{1}$ Radiology and Molecular Imaging, ${ }^{2}$ Pathology, and ${ }^{3}$ Surgery, Xijing Hospital, \\ Fourth Military Medical University, Xi'an 710032, P.R. China
}

Received January 20, 2014; Accepted March 4, 2015

DOI: $10.3892 / \mathrm{mco} .2015 .537$

\begin{abstract}
Regardless of the controversial pathogenesis, intracranial meningeal hemangiopericytoma (M-HPC) is a rare, highly cellular and vascularized mesenchymal tumor that is characterized by a high tendency for recurrence and extraneural metastasis, despite radical excision and postoperative radiotherapy. M-HPC shares similar clinical manifestations and radiological findings with meningioma, which causes difficulty in differentiation of this entity from those prognostically favorable mimics prior to surgery. Treatment of M-HPC, particularly in metastatic settings, remains a challenge. A case is described of primary M-HPC with recurrence at the initial and distant intracranial sites and extraneural multiple-organ metastases in a 36-year-old female. The metastasis of M-HPC was extremely extensive, and to the best of our knowledge this is the first case of M-HPC with delayed metastasis to the bilateral kidneys. The data suggests that preoperative computed tomography and magnetic resonance imaging could provide certain diagnostic clues and useful information for more optimal treatment planning. The results may imply that novel drugs, such as temozolomide and bevacizumab, as a component of multimodality therapy of M-HPC may deserve further investigation.
\end{abstract}

Correspondence to: Dr Juntao Han, Department of Surgery, Xijing Hospital, Fourth Military Medical University, 15 Chang Le Western Road, Xi'an 710032, P.R. China

E-mail: radiologic@163.com

Dr Hong Yin, Department of Radiology and Molecular Imaging, Xijing Hospital, Fourth Military Medical University, 15 Chang Le Western Road, Xi'an 710032, P.R. China

E-mail: bigwei@fmmu.edu.cn

Key words: magnetic resonance imaging, computed tomography treatment, brain tumor, meningeal hemangiopericytoma, metastasis

\section{Introduction}

Similar to the hemangiopericytoma of soft tissues in pathological features, first reported by Stout and Murray (1), intracranial meningeal hemangiopericytoma (M-HPC) is a rare mesenchymal tumor, possibly of pericytic origin in the meninges, which was initially described by Begg and Garret (2) in 1954. The study by Cushing and Eisenhardt (3) was the first to report a dural-based hemangiopericytoma, which was described as a variant of meningioma. M-HPC, constituting $~ 0.4 \%$ of all the primary central nervous system tumors, is a distinctive, well-defined clinicopathological entity characterized by a propensity for local recurrence and extraneural metastasis (4). In the 2007 World Health Organization Classification of Tumors of the Central Nervous System, M-HPC was identified as a distinct entity in the group of mesenchymal non-meningothelial tumors (4).

M-HPC shares similar clinical manifestations and radiological findings with meningioma and the newly recognized solitary fibrous tumor of the meninges, which makes it difficult to differentiate this entity from those prognostically favorable mimics prior to surgery. Preoperative detection and identification of M-HPC is important for improved clinical risk stratification, more optimal selection of therapy, and improved treatment response prediction and prognosis evaluation. Regardless of an enhanced understanding of the aggressive biological behavior of this type of tumor, the treatment of M-HPC remains a great challenge.

In the present study, a histopathologically and immunohistochemically confirmed case is described of M-HPC with recurrences at the primary and distant intracranial sites and extraneural metastases to multiple organs. The radiological features and treatment outcome is summarized.

\section{Case report}

Patient. A 36-year-old female presented with a 3-year history of weakness and numbness of the left lower limb and a recent headache. The patient had no history of trauma or fever. The neurological examination revealed that power was decreased in the left lower limb (grade III) when compared to the right 
Table I. Immunohistochemical features of M-HPC.

\begin{tabular}{|c|c|c|c|c|c|c|c|c|c|}
\hline Specimen & Vim & CD34 & CD99 & bcl-2 & XIIIa & S-100 & EMA & GFAP & $\mathrm{Ki}-67(\%)$ \\
\hline Primary & ++ & + & + & + & ++ & - & - & - & $8-15$ \\
\hline Recurrence & + & + & ++ & + & $\mathrm{N} / \mathrm{A}$ & - & - & - & 15 \\
\hline Metastase & + & + & ++ & - & $\mathrm{N} / \mathrm{A}$ & - & - & - & 10 \\
\hline
\end{tabular}

M-HPC, intracranial meningeal hemangiopericytoma; Vim, vimentin; EMA, epithelial membrane antigen; XIIIA, Factor XIIIa; GFAP, glial fibrillary acidic protein; N/A, not available.

lower limb (grade IV). Atrophy of the muscles of the limbs was found. The sensory system examination revealed a decrease in the sensations of the left lower limb. The laboratory findings were normal.

Computed tomography (CT) of the head revealed a right frontal isodense mass adjacent to the falx cerebri without calcification, however, minimal peritumoral edema was observed. Magnetic resonance imaging (MRI) demonstrated a large extra-axial dural-based tumor $(5.8 \times 5.2 \times 4.2 \mathrm{~cm})$ in the right frontal region. On T2-weighted imaging, the mass exhibited a predominant isointensity with minimal peritumoral edema and mass effect extending across the midline (Fig. 1A). On T1-weighted imaging, the lobulated lesion demonstrated a mixed intensity in comparison to the surrounding brain (Fig. 1B). Intense inhomogeneous contrast enhancement with cystic components and lobulated contour of the mass were noted on contrast-enhanced MRI (Fig. 1C). A possible diagnosis of M-HPC was made. Preoperative embolization at the time of cerebral angiography was used to reduce blood loss and brain injury during the surgery.

Immunohistochemical staining. Tissue blocks of the primary and recurrent tumors and the biopsy bone specimen (right ilium) were available for histopathological and immunohistochemical studies. Hematoxylin and eosin (H\&E) stains were routinely performed. Immunohistochemical stains were performed with the Dako EnVision System (Peroxidase, DAB; Dako North America, Inc., Carpinteria, CA, USA), and the following antibodies were used for immunohistochemistry: Vimentin (1:100, V9; DakoCytomation, Glostrup Denmark), glial fibrillary acidic protein (1:400, 6F2; Antibody Diagnostics, Stanford, CT, USA), cluster of differentiation (CD34) (1:100, QBEnd10; Immunotech, Marseille, France), epithelial membrane antigen (1:100, E29; DakoCytomation), S-100 (1:50, 4C4.9; rabbit polyclonal; DakoCytomation), factor XIIIa (1:500; Calbiochem, San Diego, CA, USA), CD99 (1:100, clone O13; Signet Laboratories, Dedham, MA, USA), B-cell lymphoma 2 (bcl-2) (1:200; DakoCytomation) and Ki-67 (1:50; MIB-1, DakoCytomation). The immunohistochemical results were graded subjectively according to extent as negative (-), focal $(+)$ or diffuse $(++)$.

Gross total resection was performed and histopathological examinations of the primary M-HPC revealed a typically cellular tumor composed of round to slightly spindled cells in a jumbled arrangement (Fig. 1D). Additionally, the characteristic 'staghorn' vascular pattern was revealed. Calcification was not demonstrated as epithelioid features were distinctly absent. No high-grade HPC feature was defined, and all the features were compatible with M-HPC. The immunohistochemical staining revealed positive staining for vimentin (diffuse), factor XIIIa (diffuse), CD99 (focal), bcl-2 (focal) and CD34 (focal), and negative for epithelial membrane antigen, glial fibrillary acidic protein and S-100 protein. The proliferation index evaluated with antibody against Ki-67 antigen reached 8-15\% (Table I). Single-dose, image-guided radiosurgery to the tumor bed was undertaken. The symptoms were resolved following surgery.

Follow-up. Four years later, a follow-up MRI revealed a local relapse within the original site (Fig. 2A and B) and two recurrent tumors at the right parasellar region (Fig. 2C) and right occipital region (Fig. 2D), respectively. A second total-resection surgery was performed. The resected-recurrent tumor shared similar histopathological features with the proliferation index of $15 \%$ in comparison to the primary tumor.

Series MRI and CT scan performed later disclosed multiple extracranial metastases to the ilium, costal bone, bilateral kidneys and spine with compression fractures of $\mathrm{C} 4$, T4 and T6 (Fig. 3A-G). The corresponding coronal volume-rendered single photon emission-CT images showed multiple areas of increased tracer uptake. CT-guided aspiration of the mass in the left ilium was performed and histological examination revealed a spindle cell tumor compatible with an M-HPC, with proliferation index of $10 \%$. The immunohistochemical findings of the primary, recurrent tumors and metastases are summarized in Table I.

The patient received two cycles of chemotherapy with oral $150 \mathrm{mg} / \mathrm{m}^{2}$ temozolomide on days $1-7$ and $15-21$ and $5 \mathrm{mg} / \mathrm{kg}$ bevacizumab intravenously on days 8 and 22, repeated at 28-day intervals. Two months later, in the absence of unacceptable toxicity, the patient continued to receive treatment with $4 \mathrm{mg}$ zoledronic acid once every 3 weeks for 10 cycles to prevent skeletal relevant events and to palliate bone pain. At the end of the treatment, stable disease was obtained and it lasted over one year from then on. Currently, the patient continues to be clinically and radiographically stable on MRI and CT.

\section{Discussion}

Intracranial M-HPC is a rare, but distinct highly cellular and vascularized mesenchymal tumor with a high recurrent rate of $>91 \%$ after 15 years (5) and high metastasis rate of 64\% (6). Bone, liver, lung, central nervous system and abdominal cavity are the most commonly reported sites of metastasis in HPC. The present study described a case of primary M-HPC with 

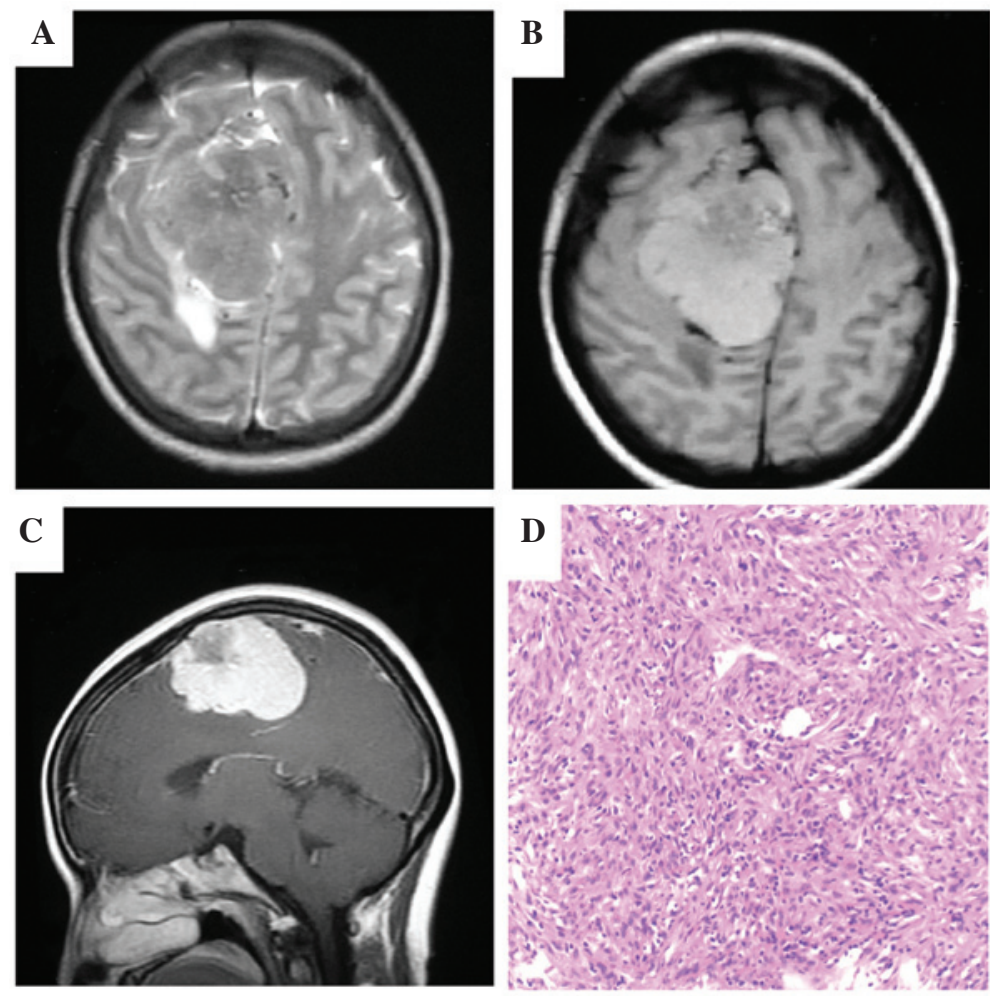

Figure 1. MRI and histopathological findings of primary meningeal hemangiopericytoma. (A) Axial T2-weighted; (B) T1-weighted; and (C) contrast-enhanced sagittal T1-weighted MR images show a mass located in the right frontoparietal parasagittal convexity. (A) The mass is isointense to gray matter on the T2-weighted MR image with moderate surrounding edema and mass effect. A CSF cleft is apparent. (B) The T1-weighted image shows an irregular parasagittal mass of heterogeneous intensity. (C) On the contrast-enhanced sagittal T1-weighted image, a marked enhancement is apparent with cystic components and narrow-based dural attachment. No typical 'dural tail sign' can be observed. (D) Hematoxylin and eosin stain reveals the pathology of the tumor is a diagnosis of meningeal hemangiopericytoma. MRI, magnetic resonance imaging; CSF, cerebrospinal fluid.
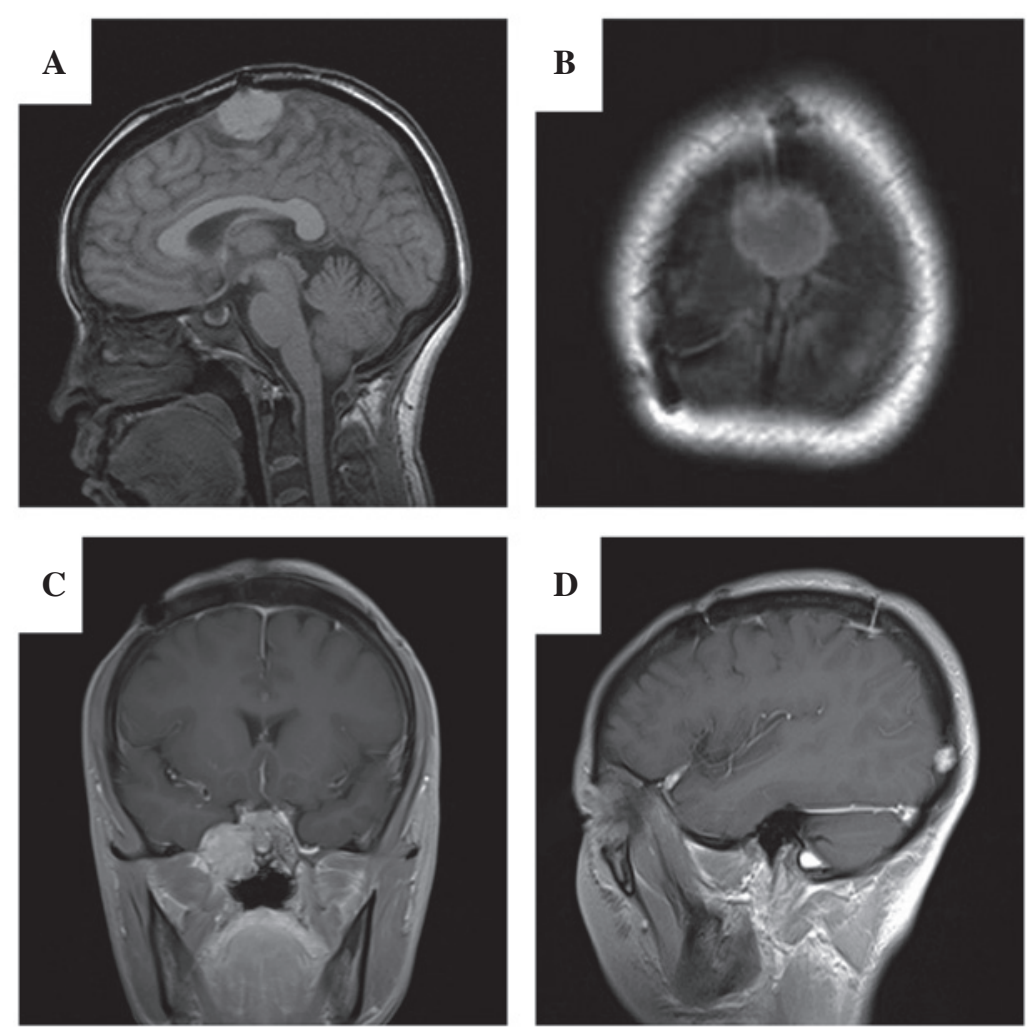

Figure 2. MRI manifestations of recurrent meningeal hemangiopericytoma at the primary and distant sites. (A) Sagittal T1-weighted; and (B) axial T1-weighted MR images show a midline trans-superior sagittal sinus mass of slight hyperintensity with a broad-based dural attachment. (C) Coronal contrast-enhanced T1-weighted MR image shows a marked enhancing extra-axial mass at the right sphenoid wing; and (D) sigittal contrast-enhanced T1-weighted MR image reveals a minor focus in the right occipital region. MRI, magnetic resonance imaging. 

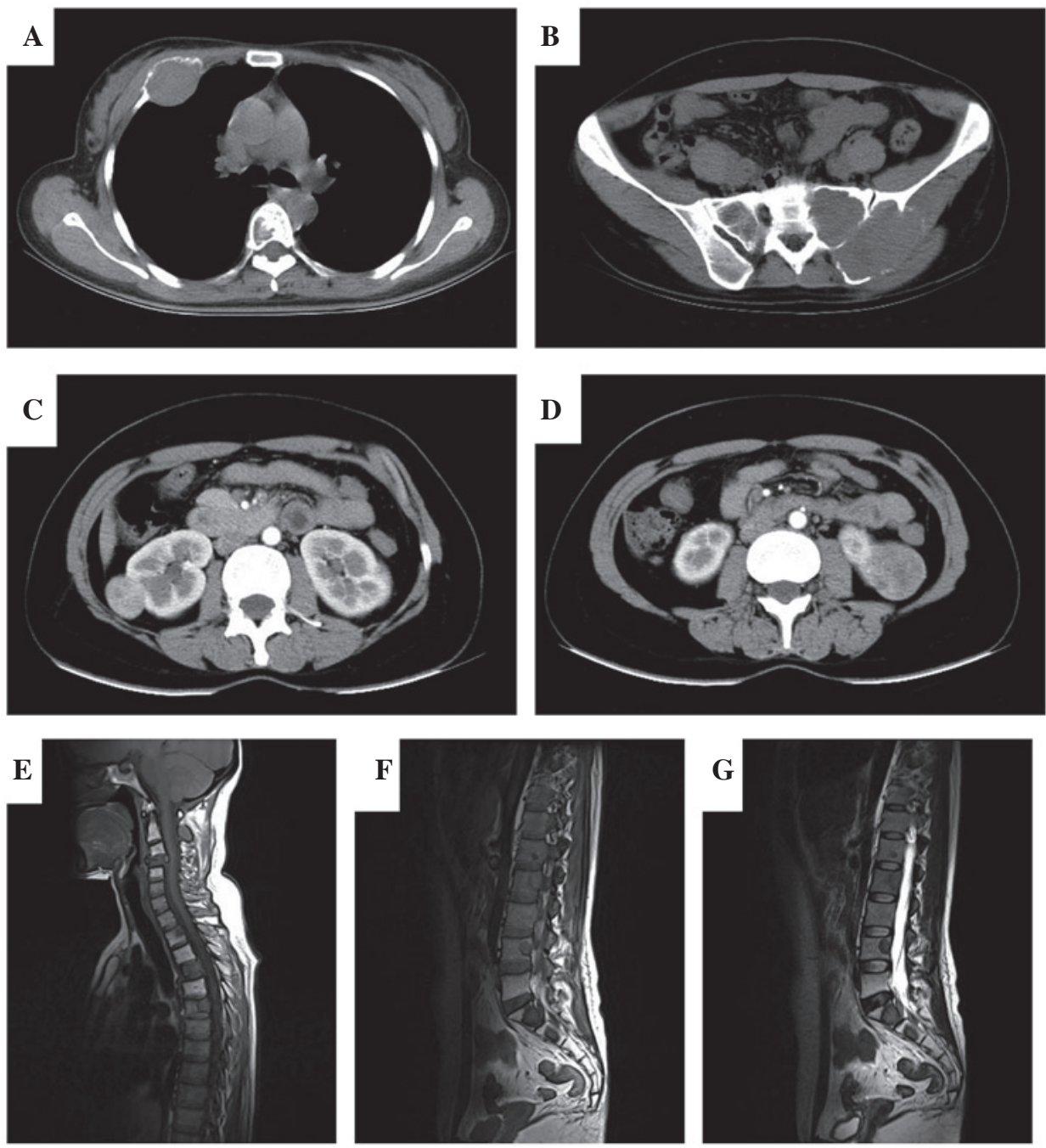

Figure 3. MRI finding of the extracranial metastasis to the bone and bilateral kidneys. (A) Axial computed tomography (CT) scan shows an expansile osteolytic lesion with a sclerotic margin in the right third rib. (B) Axial CT scan demonstrates a similar mass in the left ilium and left ala of sacrum, respectively. Axial contrast-enhanced CT scans reveal (C) bilateral enhancing renal masses in the interpolar region of the right kidney and (D) the inferior pole of the left kidney. Sagittal MRI of whole spine identifies the presence of multiple metastases in vertebral bodies, with compression fractures of C4, T4 and T6; (E) T1WI, (F) T1WI and (G) T2WI, respectively. MRI, magnetic resonance imaging; T1WI, T1-weighted image; T2WI, T2-weighted image.

recurrence at the initial and distant intracranial sites and extraneural multiple-organ metastases in a 36-year-old female. The metastasis of M-HPC was extremely extensive, and to the best of our knowledge, this is the first case of M-HPC with delayed metastasis to the bilateral kidneys.

Preoperative detection and identification of M-HPC is important with regards to therapeutic and prognostic value due to its more aggressive biology, which is distinct from that of meningioma. Clinically and radiographically, it is often challenging for differential diagnosis of M-HPC from meningioma. However, multimodality imaging, such as CT and MRI, can demonstrate the important characteristics of these tumors and may provide certain diagnostic clues. Clinically, M-HPC typically occurs at a younger age than meningiomas, and slightly more often in males compared to females (4). The clinical course of M-HPC is often shorter than that of meningiomas due to its faster growth rate. Radiographically, M-HPC is usually a sharply demarcated extra-axial mass with dural attachment, multilobulated margin and marked contrast enhancement on CT and MR imaging $(7,8)$. On T1-weighted imaging, this lesion is isointense to slightly hyperintense, which may have resulted from the nature of hypercellularity and hypervascularity. In the study by Chen et al (8), all the eight cases of M-HPC demonstrated multiple signal-intensity voids of vessels on MRI. As opposed to meningioma, M-HPC may present adjacent bony erosion, but lacks calcification and hyperostosis of the involved bone, which is indicative of meningioma. The present case represented several imaging features that are suggestive of the diagnosis. Additionally, this case clarified the requirement for detailed staging and long-term follow-up. However, these radiological profiles are not sufficiently distinctive to permit the exclusion of meningioma. The correct diagnosis primarily relies on histological and immunophenotypical confirmation.

The total removal of the tumor followed by postoperative-adjuvant radiotherapy is the mainstay of treatment. Postoperative-adjuvant radiotherapy has been reported to be effective in local-recurrence control (6,9-13), although controversy exists in its associations with the reduction of metastasis development (9) and survival benefit (13-16). 
The propensity of M-HPC in producing metastases in extraneural organs is the principal cause of failure in the treatment. The development of M-HPC metastasis resulted in a significant reduction in the survival time with an average survival time of 24 months after discovery (6). The role of chemotherapy in the treatment of the metastatic M-HPC remains controversial with varied responses in an extremely limited number of studies $(9,14,17,18)$. Certain novel drugs, including anti-vascular endothelial growth factor receptor drugs (19-21) and a tyrosine kinase inhibitor (22), have been initiated to treat this disease. Temozolomide has demonstrated activity against numerous types of cancers (23-25). In the present case, multiple metastases were discovered in multiple organs, including rib, ilium, spine and the bilateral kidneys with vertebral compression fractures. Palliative radiation therapy, with a dose of $40 \mathrm{~Gy} / 10$ fractions, resulted in alleviation of the involved bone pain. Four cycles of chemotherapy with temozolomide and bevacizumab were initiated, followed by 10 cycles of zoledronic acid. The patient continued to be clinically and radiographically stable on follow-up MRI and CT. The case provides evidence that a multimodality approach of systemic therapy with temozolomide and bevacizumab, in combination with palliative radiation therapy, may be a promising therapeutic strategy when metastatic M-HPC is encountered. However, limited to the rarity of the condition and available data reported previously, the optimal systemic treatment strategy has not been defined.

In conclusion, M-HPC shares similar clinical manifestations and radiological findings with meningioma, but it is a rare, distinct clinicopathological entity with high metastatic potential and tendency for aggressive-local recurrence. Preoperative CT and MRI could provide certain diagnostic clues and useful information for more optimal treatment planning. However, the treatment of M-HPC, particularly in metastatic settings, remains a challenge. Novel drugs, including temozolomide and bevacizumab, as a component of multimodality therapy, may deserve further investigation. Increasing the knowledge regarding the nature of this entity, underlying molecular pathogenesis and affected signaling pathways makes molecularly-targeted therapy of this lesion possible.

\section{Acknowledgements}

The authors would like to thank Professor Fucheng Ma at the Department of Pathology, Xijing Hospital (Xi'an, China) for his help in the present study.

\section{References}

1. Stout AP and Murray MR: Hemangiopericytoma: A vascular tumor featuring Zimmermann's pericytes. Ann Surg 116: 26-33, 1942.

2. Begg CF and Garret R: Hemangiopericytoma occurring in the meninges: case report. Cancer 7: 602-606, 1954.

3. Cushing H and Eisenhardt L: Meningiomas: Their Classification, Regional Behavior, Life History, and Surgical End Results. Charles C Thomas, Springfield, IL, 1938.

4. Giannini C, Rushing EJ and Hainfellner: Hemangiopericytoma. In: WHO Classification of Tumors of the Central Nervous System. Louis DN, Ohgaki H, Wiestler OD and Cavenee WK (eds). IARC Press, Lyon, pp178-180, 2007.
5. Vuorinen V, Sallinen P, Haapasalo H, et al: Outcome of 31 intracranial hemangiopericytomas: poor predictive value of cell proliferation indices. Acta Neurochir (Wien) 138: 1399-1408, 1996.

6. Guthrie BL, Ebersold MJ, Scheithauer BW and Shaw EG: Meningeal hemangiopericytoma: histopathological features, treatment, and long-term follow-up of 44 cases. Neurosurgery 25: 514-522, 1989.

7. Chiechi MV, Smirniotopoulos JG and Mena H: Intracranial hemangiopericytomas: MR and CT features. AJNR Am J Neuroradiol 17: 1365-1371, 1996.

8. Chen Q, Chen XZ, Wang JM, Li SW, Jiang T and Dai JP: Intracranial meningeal hemangiopericytomas in children and adolescents: CT and MR imaging findings. AJNR Am J Neuroradiol 33: 195-199, 2012.

9. Dufour H, Métellus P, Fuentes S, et al: Meningeal hemangiopericytoma: a retrospective study of 21 patients with special review of postoperative external radiotherapy. Neurosurgery 48: 756-763, 2001.

10. Chang SD and Sakamoto GT: The role of radiosurgery for hemangiopericytomas. Neurosurg Focus 14: e14, 2003.

11. Kumar N, Kumar R, Kapoor R, et al: Intracranial meningeal hemangiopericytoma: 10 years experience of a tertiary care Institute. Acta Neurochir (Wien) 154: 1647-1651, 2012.

12. Rutkowski MJ, Jian BJ, Bloch O, et al: Intracranial hemangiopericytoma: clinical experience and treatment considerations in a modern series of 40 adult patients. Cancer 118: 1628-1636, 2012.

13. Stessin AM, Sison C, Nieto J, Raifu M and Li B: The role of postoperative radiation therapy in the treatment of meningeal hemangiopericytoma: experience from the SEER database. Int J Radiat Oncol Biol Phys 85: 784-790, 2013.

14. Kim JH, Jung HW, Kim YS, et al: Meningeal hemangiopericytomas: long-term outcome and biological behavior. Surg Neurol 59: 47-54, 2003.

15. Rutkowski MJ, Sughrue ME, Kane AJ, et al: Predictors of mortality following treatment of intracranial hemangiopericytoma. J Neurosurg 113: 333-339, 2010.

16. Schiariti M, Goetz P, El-Maghraby H, Tailor J and Kitchen N: Hemangiopericytoma: long-term outcome revisited. Clinical article. J Neurosurg 114: 747-755, 2011.

17. Bastin KT and Mehta MP: Meningeal hemangiopericytoma: defining the role for radiation therapy. J Neurooncol 14: 277-287, 1992.

18. Fountas KN, Kapsalaki E, Kassam M, et al: Management of intracranial meningeal hemangiopericytomas: outcome and experience. Neurosurg Rev 29: 145-153, 2006.

19. Delgado M, Pérez-Ruiz E, Alcalde J, et al: Anti-angiogenic treatment (sunitinib) for disseminated malignant haemangiopericytoma: a case study and review of the literature. Case Rep Oncol 4: 55-59, 2011.

20. Kirn DH and Kramer A: Long-term freedom from disease progression with interferon alfa therapy in two patients with malignant hemangiopericytoma. J Natl Cancer Inst 88: 764-765, 1996.

21. Domont J, Massard C, Lassau N, et al: Hemangiopericytoma and antiangiogenic therapy: clinical benefit of antiangiogenic therapy (sorafenib and sunitinib) in relapsed malignant haemangioperyctoma/solitary fibrous tumour. Invest New Drugs 28: 199-202, 2010.

22. Peters KB, McLendon R, Morse MA and Vredenburgh JJ: Treatment of recurrent intracranial hemangiopericytoma with SRC-related tyrosine kinase targeted therapy: a case report. Case Rep Oncol 3: 93-97, 2010.

23. Yovine A, Riofrio M, Blay JY, et al: Phase II study of ecteinascidin-743 in advanced pretreated soft tissue sarcoma patients. J Clin Oncol 22: 890-899, 2004.

24. Park MS, Patel SR, Ludwig JA, et al: Activity of temozolomide and bevacizumab in the treatment of locally advanced, recurrent, and metastatic hemangiopericytoma and malignant solitary fibrous tumor. Cancer 117: 4939-4947, 2011.

25. Tatar Z, Thivat E, Planchat E, et al: Temozolomide and unusual indications: review of literature. Cancer Treat Rev 39: 125-135, 2013. 